Gynecol. Invest. 1976;7:120-126

\title{
Authors' Index
}

Numbers refer to abstract numbers

$52,60,84$

III 9, 24, 26, 28, 79, 112,

Abdul-Karim, R.W. 90

Abraham, G.E. 100, 101, 102

Abrams, R.M. 10

Abu-Fadil, S. 34, 110

Adamsons, K. 20, 137

Adcock, E.W., III 42

Ahn, S. 86

Ahokas, R.A. 89

Amos, D.B. 76

Amstey, M.S. 19

Andersen, G.J. 127

Andersen, R.N. 123

Anderson, D.C. 64, 109

Antonipillai, I. 168

Arias, F. 106

Assali, N.S. 9,26,79, 112, 113

Athey, B. 136

Athey, R. 58

Atienza, M.F. 96

Ayoub, E.M. 85

Ayromlooi, J. 52, 84

Badri, M.S. 85 Baer, H. 85, 93, 99 Baker, H.A. 151 Baker, R.D. 72 Baranczuk, R.J. 38 Bard, H. 78 Barker, K.L. 41,45 Barron, D.H. 92 Bastert, G. 130 Battaglia, F.C. 13,61 Bauman, A.R. 31 Bazer, F.W. 74,91 Beck, L.R. 36 Becker, R.A. 30

Beer, A.E. 53, 80 Beling, C.G. 169 Ben-Jonathan, N. 51 Berman, I.R. 8 Bernie, B.J. 12 Bessman, S.P. 86 Beydoun, S.N. 90 Bhakthavathsalan, A. Bhatnagar, Y.M. 39 Bhatnager, R.K. 151

Bhavnani, B.R. 72 Bissonnette, J.M. 94 Blandau, R.J. 50 Bloch, E. 154 Boime, I. 54, 56

Bolognese, R. 158 Braselton, W.E. 116 Brennan, S.C. 27 Brent, R.L. 44 Brinkman, C.R

113 Brodkin, L. 14 Brody, M.J. 28, 151 Bronson, R.A. 37 Brown, J.E. 143 Buchi, K.A. 67 Buhi, W.C. 74 Burd, L. 81 Burkman, R.T. 96 Burnett, L.S. 96 Burry, K.A. 66 Butler, J.R. 150

Caldwell, B.V. 133 Calvert, M.E. 126 Canales, E.S. 82

Scientific Abstracts

Tarr, B.D. 140

Carson, B.A. 13

Caton, D. 91, 92

Cederqvist, L.L. 169 
Chand, S.P. 127

Chang, R.J. 135

Charles, D. 105

Chattoraj, S.C. 105

Chen, G. 136

Chez, R.A. 25, 27, 121

Childs, R.S. 133

Chin, C.-C. 39,49

Choate, J.W. 19

Chong, A.P. 156

Clark, K.E. 24, 28, 151

Clark, S.W. 40

Geary, R.E. 119, 120

Clements, J.A. 134

Clewell, W.H. 13

Cohn, H.C. 77

Cohn, H.E. 73

Comas-Urrutia, A. 20

Congote, L.F. 71

Conrad, S. 117

Cornet, A. 78

Cousins, L. 135

Creasy, R.K. 62, 134

Cruz, A.C. 85

Csapo, A. 75, 115

Cunningham, F.G. 104, 118

Currie, W.B. 114

Daikoku, N.H. 145 Dandavino, A. 26, 79 Davajan, V. 17 Davidson, E.C. 143 Davis, B.M. 44

Dawood, M.Y. 33 Dawson, E.B. 147, 148 Dean, J.C. 70 Debrovner, C.H. 8 Dence, J.B. 49

Devoe, L. 47 Dilts, P.V.,jr. 89 Donohue, T.M. 6

Eatherly, C. 128 Ehrenkranz, R.A. 25, 27, 121 Ehrlich, C.E. 119 Ehrlich, E.N. 29, 111

Eichorst, B.C. 12 Ellegood, J.O. 116 Erdos, T. 75 Ewaschuk, E.J. 133

Fainstat, T. 38 Febres, F. 65 Ferreira, E. 76 Fisher, D.E. 155 Fouron, J.-C. 78 Fox, H.E. 19,44

Freese, U.E. 47 Fuchs, A.-R. 153 Fuchs, F. 21, 33, 169

Gabbe, S.G. 86

Galask, R.P. 129

Gale, C.C. 50

Gall, S.A. 76

Ganguly, M. 16

Gant, N.F. 126, 127, 131, 136

Gatz, M. 154

Geisz, J. 130

German, J. 46

Gibbs, C.P. 95

Givens, J.R. 123

Gohari, P. 22 
Goldkrand, J.W. 142

Goldstein, M.H. 74

Gondos, B. 65

Gotthelf, G. 1

Gottlieb, F. 141

Gregory, G.A. 134

Gusdon, J.P.,jr. 4,97

Gusseck, D.J. 55, 70

Gyves, M.T. 149

Halbert, S.A. 50 Hamada, Y. 37 Hamilton, L.A.,jr. 27, 121 Hargis, G.K. 31 Harlow, A. 157

Hauth, J.C. 104, 131 Hayashi, R.H. 30 Head, J.R. 53 Heilbron, D.C. 150 Heinrichs, W.L. 66

Heise, E.R. 97 Herbst, G.A. 97 Herman, W.K. 89

Society for Gynecologic Investigation

122

Hickok, D. 117 Hobbins, J.C. 22 Hobel, C.J. 135 Hodgson, B.J. 48 Hoehn, H. 117 Hoff, J. 15, 18 Hohler, C.W. 19 Hung, T.T. 45 Hunt, C.E. 12

Iliescu, C. 8 Iliescu, H. 8 Illner, P. 50

Jackson, B.T. 73, 77 Joelsson, I. 137 Johnson, J.W.C. 145 Johnston, J.M. 58, 68 Jones, M.D., jr. 61 Jones, O.W. 32 Judd, H.L. 32,64, 109

Kalra, P.S. 1 Kalra, S.P. 1 Karp, L. 117 Kawagoe, S. 98 Kawahara, W. 31 Kearney, K.C. 145

Keller, D.W. 125, 128 Kelley, L.K. 6 King, T.M. 96 Kitterman, J.A. 134 Kletzky, O.A. 17 Knox, G.E. 36 Koren, Z. 154 Koszalka, T.R. 44 Kunzel, W. 52, 84

110

Lachelin, G.C.L. 34, Landefeld, T. 54 Laros, R.K.,jr. 150 Larsen, B. 129 Larsen, J.W. 103

Lasley, B. 15, 18 Latta, J. 9 Lauersen, N.H. 21 Leblanc, H. 34 Lechene, C.P. 156 LeMaire, W.J.

160 Lemons, J.A. 81

Leontic, E.A. 35 Lessard, G.M. 55 Li, T.K. 120 Lim, H.S. 145 Lin, T.J. 116 Lindheimer, M.D.

29, 111 Ling, W.Y. 160 Liu, M. 60 Lo, J.-T. 43 Longo, L.D. 11 Lowe, E.W. 105 Lumeng, L. 120

MacDonald, P.C. 58, 126, 127, 136, 140

Macri, J.N. 83

Madden, J.D. 140

Mahesh, V.B. 116

Maidman, J.E. 143

Makowski, E.L. 13,61, 81

Mancilla, R. 106

Mann, L.I. 21,52,60,83,84

Manning, P. 12

March, CM. 17

Markovetz, A.J. 129

Maroulis, G.B. 100, 101, 102

Marsh, J.M. 160

Marshall, J.R. 135

Masillamoni, C.R.M. 55

Masse, D. 157

Matalon, S.V. 12 
Mathieu, P.A. 152

McGanity, W.J. 146, 147

McKenzie, J.M. 167

McLaughlin, M. 25

McWilliams, D. 54

Meldrum, D.R. 24, 28

Merkatz, I.R. 21,46, 149

Meschia, G. 13, 61, 81

Milewich, L. 58, 126, 136

Miller, R.K. 44

Mills, T.M. 116

Mishell, D.R.,jr. 17, 98

Monif, G.R.G. 93, 99

Moore, D.E. 98

Morris, J.A. 143

Morriss, F.H., jr. 42

Moss, P.L. 152

Muechler, E.K. 159

Muhlendorf, K.I. 128

Mulay, S. 164

Scientific Abstract

123

Murayama, K. 26, 79 Murphy, B.P. 168 Myers, R.E. 20, 137

Nachtigall, L. 16 Naftolin, F. 164, 167 Nakamura, R.M. 17,98 Nelson, D.M. 6 Neuman, M.R. 149 Nicoloff, J.T. 17 Noel, S.C. 95 Nolten, W.E. 29, 111 Notelovitz, M. 10 Nwosu, U. 158

Oakes, G.K. 25,27, 121 Oliver, C. 51 Oparil, S. 29, 111

Parer, J.T. 150

Parker, C.R., jr. 140

Parmely, M.J. 80

Paton, J.B. 155

Paulson, J.D. 125, 128, 138, 139

Pavy, M. 90

Paxson, C.L. 42

Peeters, L.L. 61

Petra, P.H. 66

Petricciani, J. 63

Phernetton, T.M. 122

Phibbs, R.H. 134

Piasecki, G.J. 73, 77

Pinkus, J.L. 105

Pitkin, R.M. 31,59,69

Pociask, C. 33

Polakoski, K.L. 132, 138, 139

Pollak, M.H. 152

Porter, J.C. 51, 140 
Pritchard, J.A. 118

Prough, R.A. 136

Quilligan, E.J. 86

Raghavan, K.S. 33

Rakoff, J.S. 108

Rankin, J.H.G. 122

Reich, K.A. 44

Resko, J. 66

Resnik, R. 7

Reynolds, W.A. 31,59,69

Samaan, N.A. 2 Sanyal, M.K. 146 Sarto, G.E. 144 Satin, K.P. 133 Schiller, H.S. 50, 66 Schwarz, B.E. 58, 136 Scott, J.R. 3 Scott, R.E. 145 Seeds, A.E. 12 Seki, K. 110 Selim, M.A. 149

Selmanoff, M. 14 Sever, J.L. 103 Shapiro, M.I. 157 Sheldon, R.E. 61 Shiverick, K. 165 Siimes, A.S.I. 62 Siiteri, P. 14,65 Simpson, J.L. 46 Smith, C.H. 6 Smith, E.R. 41 Solomon, S. 71, 164, 165 Soria, J. 82 Soukini, M.A. 78 Spellacy, W.N. 74,85 Speroff, L. 133 Spitzer, H.L. 68 Stegink, L.D. 69 Steiner, R.A. 50 Steinschneider, A. 90 Stevens, V.C. 5, 158 Stiefel, H.P. 80 Strickler, R.C. 166 Sullivan, D. 159 Summitt, R.L. 46

Society for Gynecologic Investigation

Sweet, F. 40, 57 Szczesna, E. 56

Tabei, T. 66 Talo,A. 48 Taubert, H.D. 130 Taymor, M.L. 156 Thaithumyanon, P. 155 Thatcher, W. 92 Therman, E. 144 Thompson, J. 99 Thompson, R.J. 93 Thysen, B. 154 Tiwary, CM. 142

Tolis, G. 167 Tonascia, J.A. 96 Tooley, W.H. 134 Turner, A.K. 105 Turner, J.H. 63, 141 Tyson, J.E. 35

Umstot, E.S. 123

Van Geldorp, H.J. 162 VanKreel, B.K. 161 Van Leeuwen, M. 162 Van Orden, D.E. 24, 28, 151

Van Orden, L.S., III 151 Van Otterlo, L.C. 23, 163 Villalobos, H. 82 Villee, C.A. 67, 146

Wade, R.S. 70 Wagenaar, W.A. 161 Walker, A.M. 25, 27, 121 Wallach,E.E. 37, 124, 158

124

Wallenburg, H.C.S. 23, 161, 162, 163

Wallis, P. 68

Ward, D.N. 2

Warren, J.C. 39,40,43,49,125

Weiner, R. 14

Weiner, S. 124

Weiss, G. 16

Weiss, R.R. 83

Welkos, S.L. 93

Whalley, P.J. 104, 131

Whitesides, D.B. 76

Wickham, W.K. 94

Wiest,W.G. 125

Wilcox, C. 92

Wilen,R. 164

Williams, G.A. 31

Wiser, W.L. 123 
Wladimiroff, J.W. 23, 163

Wolfs, G.M. 162

Wong, W.S. 150

Woods, J.R., jr. 9,24,26,79, 112, 113

Woolever, C.A. 166

Worley, R.J. 127

Wright, K.H. 37, 124

Wu, M.L. 141

Yang, J. 110

Yang, P. 2

Yen, S.S.C. 15, 18, 32, 34, 64, 107, 108,

109,110 Young, P.C.M. 119 Young, P.E. 32

Zacur, H.A. 35 Zarate, A. 82 Zawaneh, S. 85 